\title{
Facilitators of and barriers to successful teamwork during resuscitations in a neonatal intensive care unit
}

\author{
Zeynep N. Inanc Salih ${ }^{1} \cdot$ Claire Burke Draucker ${ }^{2}$
}

Received: 23 August 2018 / Revised: 24 March 2019 / Accepted: 26 March 2019 / Published online: 16 May 2019

(c) Springer Nature America, Inc. 2019

\begin{abstract}
Objective Effective teamwork is essential in high-risk healthcare delivery environments. In this study, we aimed to identify facilitators of and barriers to successful teamwork during resuscitations in the NICU Study Design: 36 in-situ interprofessional simulation sessions were held in a level 4 NICU. Each session was followed by a debriefing where staff talked about the simulation scenario but also about their prior experiences during resuscitations in the NICU. Using content analysis, we analyzed the transcriptions of debriefings to address the study aims.

Result Participant responses yielded three major themes: communicating well, getting tasks done well, and working well together. Each main theme had subthemes.

Conclusion Teamwork is a complex process that is enhanced and hindered by a variety of factors. The factors identified in this study can be used to enhance relationship-based teamwork training programs. Future research is needed to determine which teamwork behaviors are most associated with patient outcomes
\end{abstract}

\section{Introduction}

Healthcare delivery in high-risk situations can be best accomplished by teams with good teamwork skills [1]. Following the Institute of Medicine reports on patient safety and medical errors (i.e., "To Err is Human" and "Crossing the Quality Chasm") there has been an enhanced focus on assessing and improving teamwork in critical care settings [2, 3] Moreover, the Joint Commission identified communication problems among healthcare providers as the leading cause of neonatal death and recommended team training in perinatal units [4].

Teamwork training in healthcare is often based on widely used programs such as crew resource management (CRM) and TeamSTEPPS (Team Strategies and Tools to Enhance Performance and Patient Safety). CRM is designed to identify threats to an operation and develop, communicate and carry out a plan to avoid and mitigate that threat [5]. The widespread use and acceptability of CRM in the

Zeynep N. Inanc Salih

zsalih@iu.edu

1 Indiana University School of Medicine, Indianapolis, IN, USA

2 Indiana University School of Nursing, Indianapolis, IN, USA aviation industry has led researchers to use CRM principles to develop team training programs for healthcare teams. However, a program directly adopted from the aviation industry may not be suitable for all healthcare teams [6]. Further, the Agency for Healthcare Research and Quality (AHRQ) questioned the effectiveness of CRM in improving patient safety due to lack of evidence of outcomes in both medicine and aviation [7]. TeamSTEPPS was developed by the Department of Defense Patient Safety Program in collaboration with AHRQ [8]. This program focuses on communication, leadership, situation monitoring and mutual support. While both training programs emphasize similar teamwork skills, they may not meet the needs for team training in a variety of healthcare settings and for teams performing specific tasks [9]. For example, it is unclear if these programs would be applicable to training staff in a neonatal intensive care unit (NICU) to conduct resuscitations on newborns. Some experts recommend that each service in healthcare institutions should develop and test its own core teamwork competency taxonomies in order to develop context-specific teamwork training [6].

Despite the importance of successful teamwork in neonatal resuscitation, little is known about factors that influence how interprofessional teams work together during this acute situation [10]. Hunziker et al. [11] argued that despite substantial efforts to make cardiopulmonary resuscitation 
(CPR) algorithms known to healthcare workers, the outcome of CPR has remained poor during the past decades. They propose that further studies on factors influencing team interactions, such as team member status and team hierarchy, on the performance of complex medical emergency interventions are needed. The Neonatal Resuscitation Program (NRP) was developed to teach providers how to care for newborns at birth and has been credited with improving the health of thousands of newborns worldwide [12]. In its seventh edition, the NRP identifies ten behavioral objectives to promote teamwork: (1) know the environment, (2) anticipate and plan, (3) assume leadership role, (4) communicate effectively, (5) delegate workload optimally, (6) allocate attention wisely, (7) use all available information, (8) use all available resources, (9) call for help when needed, and (10) maintain professionalism [13]. These behavioral objectives are adapted mostly from CRM principles and primarily aimed at teams in the labor and delivery rooms. Some recommendations, such as assigning roles and responsibilities, performing an equipment check, and making an action plan before a resuscitation event might not be possible in the NICU where ad hoc teams usually have to gather quickly with little or no time to plan.

A few studies have explored how staff view the essential elements of teamwork during resuscitations in medicine and in NICU. In a study by Bristowe et al. [14], staff opinions on optimal teamwork centered on good leadership and availability of experienced staff. Thomas, Sexton and Helmreich organized focus group interviews with staff working in the NICU to elicit provider perspectives on working together in the NICU [10]. These focus group interviews yielded the following three themes: (1) provider characteristics (personal attributes, reputation, and expertise), (2) workplace factors (staffing, work organizations, and work environment) and (3) group influences (communication among providers, relationships, and teams). The results of this study capture the unique complexity of the NICU environment and point to the need to identify teamwork factors that may be specific to the resuscitations in the NICU. In this study we aimed to identify facilitators of and barriers to successful interprofessional teamwork during simulated resuscitation scenarios in a NICU using qualitative methodology.

\section{Methods}

\section{Setting and participants}

Data for this study were drawn from a larger study aimed at investigating the effects of in-situ simulations and structured debriefings on teamwork during resuscitations in the
NICU. The setting of this study was a 55-bed level IV NICU in a teaching hospital for children in the Midwestern region of the United States that serves as a referral center for the state. The unit, which does not have a high-risk obstetrics unit attached, has 700 admissions in a year with the range length of stay of 1-190 days and the average length of stay 37 days. The study was conducted between February and May of 2012. At the time of the study the NICU had multipatient rooms (modules) that housed six to eight incubators. The NICU was staffed by $\sim 150$ nurses (RNs), 30 respiratory therapists (RTs), 20 neonatal nurse practitioners (NNPs), 20 neonatologists, and 9 neonatology fellows (MDs).

After all required human protections approvals were obtained, all staff members on the unit, including those on day and night shifts, were invited to participate in the study. Staff were provided a study information sheet that described the study activities and indicated that the simulations would be videotaped and the facilitated debriefing sessions would be audio and video recorded. All participants verbally consented to participate in the study. Over a two-month period, 50 staff members were recruited by personal e-mails, flyers and/or personal contact by the principal investigator (ZS). Because some study activities occurred when participants were not scheduled to work, \$50 gift cards were provided to help with possible expenses like gas, parking, and child care.

The sample included 5 physicians (MD) (all neonatology fellows), 7 NNPs, 29 RNs, and 13 RTs. Three of the participants were men (two RTs and one MD) and the rest were women, which generally represented the gender distribution of NICU personnel and NICU fellows at the time of the study. The tenure of the participants in the current NICU ranged between 0.17 and 35 years [Table 1]. No neonatologists or pediatric residents participated due to scheduling or rotation constraints. All participants had been trained with the 6th edition of NRP as was consistent with NICU policies at the time of the study.

The research team conducted 36 simulation sessions in a separate room in the NICU that was set up with all the equipment for a newborn under a warmer. A high fidelity

Table 1 Demographic characteristics of simulation session participants

\begin{tabular}{lllc}
\hline Discipline $(n)$ & $\begin{array}{l}\text { Shift (day/ } \\
\text { night/both) }\end{array}$ & Gender & $\begin{array}{l}\text { Tenure in the NICU-range } \\
\text { and average-years }\end{array}$ \\
\hline & & $\begin{array}{l}\text { Male/ } \\
\text { female }\end{array}$ \\
RN (29) & $19 / 8 / 2$ & $0 / 29$ & $0.17-35(13.25)$ \\
RT (13) & $11 / 2 / 0$ & $2 / 11$ & $5-25(15.15)$ \\
NNP (7) & $4 / 0 / 3$ & $0 / 7$ & $0.67-20(4.99)$ \\
MD (5) & $0 / 0 / 5$ & $1 / 4$ & $0.7-2.5(1.26)$ \\
\hline
\end{tabular}


manikin was used (SimNewB, Laerdal Medical Inc.). Simulation scenarios included an accidental extubation, pneumothorax on the ventilator, and prolonged hypoxia after accidental extubation with a relatively late response to resuscitation. NRP scenarios were not used because the labor and delivery scenarios of that program were not highly applicable to the NICU personnel.

Each team had an MD or NNP, one to three RNs, and one RT. Except for three nurses who requested to be on the same team, all the other staff were assigned to teams based on their availability for the day and time of the scenario. Each team participated in three different scenarios. Two scenarios occurred in two simulation sessions with the same teams on the first day. The third scenario occurred in a third simulation session 3 months later. The composition of the teams changed for the third simulation session. All simulation sessions were video recorded.

Immediately following each simulation session, a debriefing session was held with staff members who had participated together in the simulation session. Staff sat around a table and light snacks were provided. The debriefing sessions were conducted by facilitators who represented nursing, respiratory therapy, and neonatology. The facilitators had been trained in NRP, completed a training workshop on how to debrief a simulated resuscitation event, and were skilled in managing group dynamics. Video footage of the simulation session was played during debriefings as deemed appropriate by the facilitators. During the facilitated debriefings, the following four domains of the Clinical Teamwork Scale were used to structure discussions: communication, situational awareness, decisionmaking, and leadership [15].

The debriefings began with some structured questions providing the participants the opportunity to reflect on the simulation experience they had just completed. The facilitators asked the team what was happening in the scenario, what went well, and what they would do differently. Because we also wanted the simulations to serve as a catalyst for participants to relate their simulation experiences to their "real-world" teamwork experiences during resuscitations, facilitators asked participants to share any past relevant experiences with teamwork from their own day-to-day practices that stood out to them. As a result, the narratives yielded robust data based on both simulation and practice experiences needed to identify important barriers to and facilitators of teamwork during resuscitations. The debriefing sessions, which lasted 40-60 min, were audio and video recorded and transcribed verbatim. Following the debriefing sessions, participants completed surveys to evaluate their satisfaction with the sessions. Most indicated that they were highly satisfied with the sessions and, in fact, several requested more on-going sessions.

\section{Data analysis}

The data were analyzed by the first author (ZS), a neonatologist with expertise in neonatal resuscitation and simulated learning, and the second author (CBD), a nurse researcher with expertize in qualitative methods. A qualitative descriptive approach as described by Sandelowski was used to conduct the study [16]. Qualitative description is used when a straightforward low-inference description of narrative data is required to answer a practical research question.

The authors read all the transcripts several times, highlighted relevant sections of text (text units) that addressed facilitators of and barriers to successful interprofessional teamwork during neonatal resuscitations, and coded the text units with short phrases that captured the meaning of the participants' remarks. The data were organized in several matrices as described by Miles \& Huberman [17]. First, a two-variable case-ordered matrix was constructed. The cases (participants) were displayed on the vertical axis and were divided according to professional role (RNs, RTs, NNPs, and MDs). The two variables of facilitators of and barriers to successful teamwork were displayed on the horizontal axis. Codes were placed each appropriate cell. For example, codes reflecting perceived barriers to teamwork identified by RNs would be placed in the $R N x$ Barrier cell in the matrix.

The two authors then met on several occasions to organize the codes in each cell and between cells into categories according to procedures discussed by Saldaña [18]. Through the processes of discussion, iterative reexaminations of the data, and consensus, three major categories and several subcategories within each category were identified. The subcategories represent distinct dimensions within each category. A second matrix was then constructed in which professional roles (RNs, RTs, NNPs, and MDs) were displayed on the vertical access and the categories/subcategories were displayed on the horizontal access and codes were placed in the appropriate cell. Data were again reexamined, categories/subcategories were refined, and narratives describing each category/subcategory were written with special attention to differences in responses among participants in the four professional roles.

\section{Results}

Participant responses to questions about the factors that would facilitate a good teamwork during resuscitations in the NICU centered around three major themes: communicating well, getting tasks done well, and working well together [Table 2]. 
Table 2 Facilitators of and Barriers to Successful Teamwork in the NICU

\begin{tabular}{lll}
\hline Speaking out loud & Getting task done well & Working well together \\
\hline Speaking out loud & Having clear tasks to focus on & Working synergistically \\
Sharing thoughts & Having tasks well coordinated & Managing conflict \\
Clarifying what is heard & Having the right team to do the tasks & \\
\hline
\end{tabular}

\section{Communicating well}

The participants indicated the most important factor underlying successful teamwork during a code in the neonatal unit is communicating well. This included three processes that we have labeled saying it out loud, sharing one's thoughts, and clarifying what one hears.

\section{Saying it out loud}

Many of the participants suggested that saying it out loud is an important aspect of communicating well during a code. Saying it out loud includes vocalizing what one is thinking, doing, or intends to do. One RT described this behavior as "saying out loud what one is thinking on the inside." Saying it out loud includes reporting the neonate's vital signs, the team member's action (e.g., counting chest compressions, meds given), and the progress of the code (e.g., how long the neonate has been "down"). The participants stressed that it was particularly critical that the team leader, the recorder, team members working on the "periphery" of the code, and team members who could not see the monitor receive information from team members. One RN discussed the importance of the "quick tell all"-providing new team members who join the code a brief synopsis about what had gone on in the code to that point.

Several factors, including the volume, frequency, and tone of team members' voices, influenced whether saying it out loud contributed to effective teamwork. Several participants complained, for example, that team members often do not speak loud enough. One RN advised that "soft spoken people need to speak up." Conversely, some participants complained that some members speak too loudly; one RN called this "freak out loud talking." The participants disagreed somewhat on the frequency with which team members should "say it out loud." Several cautioned against repeating vital signs too frequently. For example, an $\mathrm{RN}$ revealed that repeating the heart rate too frequently makes the team member who is intubating nervous and a physician remarked that some team members who repeat vital signs "inappropriately" are being "passive aggressive" as they are indirectly suggesting the leader is doing something "stupid." Several participants suggested that it is best if team members report vital signs only when there is a significant change in a neonate's status. A few participants suggested that the tone of team members' voice when saying it out loud was important. An RN stated, "Saying sats (saturations) calmly and gently is reassuring."

\section{Sharing one's thoughts}

Many of the participants also suggested that sharing one's thoughts is an important aspect of communicating well during a code. Whereas saying it out loud involves reporting pertinent information, sharing one's thoughts involves expressing one's opinions. One RN, for example, indicated that it is important for team members to "speak out if a code is not going in the right direction." Sharing one's thoughts can include providing others, including the team leader, with recommendations and disagreeing with others' actions during the code. Barriers to sharing one's thoughts include team members' lack of experience or being new to a team, non-assertiveness, cultural prohibitions against disagreeing with those in positions of authority, and lack of confidence in one's views. Participants indicated that some team members are reluctant to share one's thoughts if giving feedback is perceived as "running the show." Teams that "give everyone permission to express their ideas" and that encourage new team members to speak up facilitate the sharing of one's thoughts. As with saying it out loud, the tone of sharing one's thoughts affects how the message is received, and participants recommended that feedback should be given in a way that is "nice and low-toned" rather than harsh.

\section{Clarifying what one hears}

A few participants indicated that communicating well during a code also includes clarifying what one hears, which is providing feedback to those who share information or express their opinions so that there is "back and forth" communication. This process can include repeating what was said, confirming that a team member has been heard, checking one's observations with other team members, and clarifying if something was said that was unclear. The participants referred to this process as "closing the loop." A barrier occurs when team members, especially the team leader, is so focused on a task that they did not answer questions posed to them or did not respond to comments by team members. 


\section{Getting tasks done well}

The participants indicated that another important factor underlying successful teamwork during a code in the neonatal unit is Getting Tasks Done Well. This process was based on how the team's work was structured. Getting Tasks Done Well included three processes that we have labeled having clear tasks to focus on, having the tasks well coordinated, and having the right team to do the tasks.

\section{Having clear tasks to focus on}

Many participants suggested that having each team member responsible for a specific task was essential to a successful code. The participants discussed the importance of "dividing tasks," and "delineating roles." They listed a number of tasks that they believed should be assigned to specific staff members including doing chest compressions, "bagging," administering medications, managing equipment, recording, "running" (e.g., for needed medications and equipment), watching the monitors, and standing back and seeing the "whole picture."

Several negative outcomes were associated with not having clear tasks to focus on. Several participants mentioned that without assigned roles, team members feel awkward and useless as they "stand around" waiting to be told what to do. In addition, without assigned roles, team members may be required to "multi-task." RTs and physicians in particular stressed that team members could be distracted if they had to perform two tasks at once (e.g., giving medications and recording), had to do a task while directing the code (e.g., doing compressions and giving orders), or had to "do" and "think" at the same time.

The participants brought up some of the complexities of making sure that all team members have a clear task to focus on and provided some suggestions about how this might happen. For example, a couple of participants mentioned that staff who have been working together for a long time "self-identify" the roles they will perform in a code, and this becomes a problem for new staff members. Several participants advised assigning roles prior to beginning of a code so all team members, even those who are new, are clearly aware of what they should do. The participants stressed that the experience level of the team members should determine how tasks are assigned. One RN, for example, advised that new nurses should not be assigned to be "runners" or "recorders." A few participants recommended assigning an "inside circle" to do hands-on tasks "at the bedside," and an "outside circle" to "see the whole picture" and direct the code. One physician recommended having separate "functional" teams within the larger team, such as a chest compression team, a respiratory team, and an IV team.

\section{Having the tasks well coordinated}

Many participants stressed the importance of having the tasks well coordinated by a clearly designated leader. Several participants mentioned, for example, that the code nurse or the charge nurse should be the one to assign roles and facilitate the functioning of the whole team. Others indicated that the physician or NNP who was directing the code should be the leader. Many of the participants pointed to frustration that arises when there is not a clear leader or when more than one person gives orders. A few RNs, for example, revealed that teams can experience confusion over whether RNs need to wait for the physician or the NNP to "give the okay" to start chest compressions or other tasks.

The participants identified several leadership behaviors that facilitate the coordination of the tasks including being specific and direct in their instructions. For example, one $\mathrm{RN}$ pointed out the importance of "tying someone's name to the task" rather than just calling out what needs to be done. A few participants stressed that a good leader should accept input from team members and set a calm tone. One RN stated, "[The] leader panics, everyone panics."

\section{Having the right team to do the tasks}

In addition, to have well-designated tasks and good leadership, participants also stressed that having the right team to do the tasks was essential to a successful code. The right team included having the right number of team members. Many of the participants agreed that having too many people at a code-i.e., "being bombarded by bodies"caused confusion. The participants shared examples of not being able to get close enough to the patient to give meds or having too many team members report the heart rate at once. Conversely, being "short-handed" is also a problem as team members are then forced to multi-task.

The participants also indicated that having the right team involved having the right "mix" of team members. Having too many inexperienced staff, for example, could be a problem. Participants indicated that inexperienced staff members do not have "that forward thinking," tend to think in "black and white," and do not trust their own skills. Teams with too many new staff were referred to as a "weak mix."

The participants provided several suggestions about having the right team at a code. A few suggested that teams should be able to ask team members to leave the code if there are too many staff present and should have a procedure for asking for additional help if needed. Several recommendations were also made about inexperienced staff at codes. Several nurses and RTs suggested that inexperienced members could be paired with experienced staff or assigned to observe codes. 


\section{Working well together}

The participants indicated that a third important factor underlying successful teamwork during a code in the neonatal unit is working well together. This process includes instead interacting well as teammates. Working well together included two processes that we have labeled "working synergistically" and "managing conflict."

\section{Working synergistically}

Participants indicated that codes were most successful when the teams were working synergistically-that is, all the members were "in sync" with each other and the group was cohesive. According to several of the participants, this occurs when team members trust each other, have confidence in each other, and have worked together before. The participants suggested that members of such teams could anticipate each other's needs, "swap in and out" with each other when need be, take for granted that things would be done well, and rely on one another. Team synergy was associated with a feeling of being "close to" and "supported by" others on the team. One nurse explained, "You've worked together so long you can almost read each other's mind."

Several things could interfere with a team working synergistically. Having new persons join the team, for example, could upset the synergy. Several participants suggested that problems arise when "you don't know your teammates" or when team members were on "separate avenues." One NNP mentioned that teams experience problems if new team members come from a unit with a different "culture." Functioning as a hierarchy, such as when team members are intimidated by those leading the team, also interfered with synergy.

\section{Managing conflicts}

Participants indicated that managing conflicts was an important part of working well together. They stressed that such conflicts would often arise during codes and successful teams therefore need to manage these conflicts. Examples of interpersonal conflicts revealed by the participants included team members being "shut down" when they made suggestions, team members harshly judging others' performances or decisions, team members being offended if their actions were questioned, and team members appearing angry when they might not be. One RT gave the example of nurses "elbowing" RTs out of the way to do the "bagging." Interpersonal conflicts were exacerbated if the leader was insensitive to team members' feelings. "Finger pointing" and blaming others were most common when the code was not going well.
The participants believed that the best way to handle interpersonal conflict was to "debrief" after the code was over. They suggested that debriefing prevents "resentments" from festering and allows teams the opportunity to figure out how they could work better together. Several suggested that resolution of interpersonal conflicts requires that team members be willing to talk about what troubled them and ask others what bothered them if they seemed upset during a code. One RT suggested that a good debriefing allows one to "talk about an event, feel better, let it go, and move on."

\section{Discussion}

In this study, reviewing the videos of the simulated resuscitations served as a valuable springboard that sparked discussions of facilitators of and barriers to successful teamwork during resuscitations in the NICU. During these discussions, participants identified three interrelated processes that were essential for successful teamwork: communicating well, getting tasks done well, and working well together.

Our participants indicated that communicating well was the most important element of teamwork. The elements of communicating well identified by our participants resonate with some prior studies, although a variety of labels are used to describe these elements. For example, saying it out loud has also been referred to in the teamwork literature as information sharing $[19,20]$ or orientation of new members [15]. Reporting vital signs and informing other team members, especially new persons joining the code, have been identified as clear drivers of team performance $[10,21]$. Some studies found that information sharing is an important skill for reducing neonatal resuscitation duration and improving team performance [19]. Similar to our findings on sharing one's thoughts, other studies found team performance and patient safety are compromised when team members do not share what they think needs to be done [19, 22-24]. Some studies found that team members who lacked experience or confidence and who were nonassertive, and those who worked on teams with rigid authority differentials, had difficulty freely expressing their thoughts and ideas [22, 25]. Clarifying what one hears or "closing the loop" has been frequently discussed in the literature as an important skill, especially in task-based teamwork training [26, 27].

Our findings related to getting the tasks done well and working well together were also in line with some extant literature. Other studies also found, for example, that having a right number of team members with adequate skills and experiences and having well-defined roles were important to teamwork $[10,28]$. Just as our participants pointed to the importance of having tasks well coordinated, in a recent 
focus group study among emergency teams, leaders who communicate clear objectives and delegate roles and tasks equally are considered to be engaged in directive leadership [14]. Similarly, just as our participants indicated that leaders should attend to team members input, one study demonstrated that flexible leadership in ICU teams was associated with successful task completion [29].

While there is extensive literature on the importance of conflict management in a variety of settings [30, 31], our finding about the importance of managing conflict as part of teamwork during CPR has not been addressed adequately in prior studies [32-35]. Just as our participants provided examples of instances when team members were uncivil to each other during codes, research has shown that rudeness among team members has adverse consequences on diagnostic and procedural performance in the NICU [36]. Based on focus group feedback, Patterson et al. [34] added a measure of team conflict to their survey measuring teamwork among emergency medical technicians. Just as our participants remarked on the emotional ramifications of team conflict, Bristowe et al. [37] recommended addressing the handling of emotions and poor nonverbal communication in resolving conflicts in medical emergencies [14]. Like our findings, other studies have also revealed that informal debriefings and discussions were perceived to be the best means of resolving team conflicts.

Given that all participants had NRP training, it was important to consider why so many teamwork issues remained. One possible explanation is that at the time of our study, participants were trained using 6th edition of NRP, which did not include the ten teamwork behaviors by CRM. However, some issues such as conflict resolution and attending to the emotional needs of staff are not the focus of 7th edition of NRP either. In addition, NRP does not focus on teamwork issues that arise specifically in the NICU. Most importantly, we feel that teamwork issues brought to light in our study may be related to the lack of sociological fidelity [38] involved with NRP training; that is, the teams that perform actual resuscitations are not necessarily trained together and issues such as team hierarchy or interprofessional conflict are not addressed. Therefore, the training does not allow for the building of relationships or the resolution of conflicts specific to a team. More research is needed to determine differences in teamwork outcomes between interprofessional teams trained together versus training that occurs within single disciplines.

While our study supports many findings in prior research, this work contributes to the literature in several ways. First, to our knowledge, this is the first study exploring staff perceptions of teamwork for successful resuscitations in the NICU by using debriefings after in-situ simulations. Because our participants had just participated in a simulated code and were encouraged to express their thoughts freely, they provided some robust "in the moment" thoughts and intense feelings about what helps and hinders successful teamwork during a resuscitation in the NICU. Secondly, almost all of the published teamwork studies reported using either students or uniprofessional teams, whereas our participants were interprofessional colleagues who worked regularly together in the same NICU. Accordingly, they were able to bring many examples from "real-life" interprofessional experiences that contributed to the richness of the data.

Our findings should be understood in the context of some study limitations. Because the findings were based on the participants' experiences with the simulation scenarios and actual resuscitations, it is impossible to attribute findings specifically to either type of experience. However, we believe our broad study aims-identifying teamwork facilitators and barriers-were best served by using the simulation experiences to spur discussions about the participants' experiences of facilitators and barriers more generally. An optimal study design would be to video record actual resuscitations, record debriefings that follow those, and compare teamwork issues that rise in simulations versus in real practice. Another study limitation was related to common restrictions that stem from gathering data in groups. This procedure might have resulted in some participant voices not being well represented in the findings if these participants were reticent or disengaged. For example, participants new to the unit might have been reluctant that bring up adverse experiences they had with teamwork. We attempted to minimize this problem by using well-trained leaders who used basic focus group techniques to encourage engagement by all participants. Another limitation was that no attending neonatologist participated in the scenarios and thus their perspectives are not represented in the findings. However, on our unit, fellows regularly run resuscitations in the NICU along with the attending neonatologists and thus the fellows provided key perspectives on the experience of team leadership. Lastly, this study was conducted in one NICU in one hospital, which may limit the generalizability of the findings. Ultimately, studies need to be designed that investigate which teamwork behaviors in a variety of settings are most associated with patient outcomes.

Despite these limitations, our findings do have some practice implications. Knowledge about the barriers to and facilitators of teamwork that we have identified, because they are grounded in experiences of "real-life" team members who conduct resuscitations in the NICU, can be incorporated into team building trainings conducted in NICU settings. Teams could be presented with our findings and be encouraged to discuss what gets in the way of-or what helps with-the processes of communicating well, getting tasks done well, and working well together during resuscitations in their own NICU. For example, team 
members could be encouraged to reflect upon their own behaviors of saying it out loud, sharing one's thoughts, and clarifying what one hears during codes and explore ways they might change these behaviors for more effective team performance. As a group, teams could be encouraged to evaluate, without judgment, if they routinely have clear tasks to focus on, have tasks well coordinated, and have the right team to do the tasks. For example, trainers could initiate discussions of what teams think is the ideal number and composition of team members to effectively conduct codes in the NICU in a variety of situations. Finally, our findings suggest that managing conflicts successfully is integral to successful teamwork. Team trainings might thus focus on helping teams plan debriefing sessions that are conducive to not only problem-solving for more effective performance but also attend to the emotions of team members in a safe atmosphere.

In conclusion, by hearing the voices of health professionals from a variety of disciplines who work in high stress situations, we can obtain a more nuanced understanding of the complexities of teamwork. Further research is required to explore whether a relationship-based teamwork training program targeting these identified teamwork behaviors would improve teamwork and eventually patient outcomes during resuscitations in the NICU.

Acknowledgements This study was supported by a Project Development Team within the ICTSI NIH/NCRR Grant Number TR000006. Material is original research, has not been previously published and has not been submitted for publication elsewhere while under consideration

\section{Compliance with ethical standards}

Conflict of interest The authors declare that they have no conflict of interest.

Publisher's note: Springer Nature remains neutral with regard to jurisdictional claims in published maps and institutional affiliations.

\section{References}

1. Baker DP, Salas E, King H, Battles J, Barach P. The role of teamwork in the professional education of physicians: current status and assessment recommendations. Jt Comm J Qual Patient Saf. 2005;31:185-202.

2. Institute of Medicine. To err is human: building a safer health system. A report of the Committee on Quality of Health Care in America, Institute of Medicine. Washington, DC: National Academy Press; 2000.

3. Institute of Medicine. Crossing the quality chasm: a new health system for the $21 \mathrm{st}$ century. Washington, DC: The National Academies Press; 2001. 360pp.

4. Commission TJ. Preventing infant death and injury during delivery. 2004. https://www.jointcommission.org/sentinel_event_alert_ issue_30_preventing_infant_death_and_injury_during_delivery/.
5. Crew resource management training. Washington, D.C.: US Dept of Transportation, Federal Aviation Administration; 1993. 15, 6, 1 pp.

6. Baker DP, Day R, Salas E. Teamwork as an essential component of high-reliability organizations. Health Serv Res. 2006;41(4 Pt 2):1576-98.

7. Shojania KG, Duncan BW, McDonald KM, Wachter RM, Markowitz AJ. Crew resource management and its applications in medicine. 2001.

8. TeamSTEPPS ${ }^{\bullet}$ : team strategies \& tools to enhance performance \& patient safety Rockville, MD: Agency for Healthcare Research and Quality; 2018. https://www.ahrq.gov/teamstepps/ index.html.

9. Thomas EJ. Improving teamwork in healthcare: current approaches and the path forward. BMJ Qual Saf. 2011;20:647-50.

10. Thomas EJ, Sexton JB, Helmreich RL. Translating teamwork behaviours from aviation to healthcare: development of behavioural markers for neonatal resuscitation. Qual Saf Health Care. 2004;13(Suppl 1):i57-64.

11. Hunziker S, Johansson AC, Tschan F, Semmer NK, Rock L, Howell MD, et al. Teamwork and leadership in cardiopulmonary resuscitation. J Am Coll Cardiol. 2011;57:2381-8.

12. Dempsey E, Pammi M, Ryan AC, Barrington KJ. Standardised formal resuscitation training programmes for reducing mortality and morbidity in newborn infants. Cochrane Database of Syst Rev. 2015: CD009106.

13. Weiner GM, editor. Textbook of neonatal resuscitation. 7th ed. Elk Grove Village, IL: American Academy of Pediatrics, American Heart Association. Vol. xiii; 2016. 305pp.

14. Bristowe K, Siassakos D, Hambly H, Angouri J, Yelland A, Draycott TJ, et al. Teamwork for clinical emergencies: interprofessional focus group analysis and triangulation with simulation. Qual Health Res. 2012;22:1383-94.

15. Guise JM, Deering SH, Kanki BG, Osterweil P, Li H, Mori M, et al. Validation of a tool to measure and promote clinical teamwork. Simul Healthc. 2008;3:217-23.

16. Sandelowski M. Whatever happened to qualitative description? Res Nurs Health. 2000;23:334-40.

17. Miles MBHAM, Qualitative data analysis: an expanded sourcebook: Thousand Oaks: Sage Publications; 1994. http://catdir.loc. gov/catdir/enhancements/fy0655/93041204-t.html.

18. Saldaña J. The coding manual for qualitative researchers. 2nd ed. Vol. xix. Los Angeles: SAGE Publications; 2013. 303pp.

19. Katakam LI, Trickey AW, Thomas EJ. Speaking up and sharing information improves trainee neonatal resuscitations. J Patient Saf. 2012;8:202-9.

20. Thomas EJ, Sexton JB, Lasky RE, Helmreich RL, Crandell DS, Tyson J. Teamwork and quality during neonatal care in the delivery room. J Perinatol. 2006;26:163-9.

21. Mesmer-Magnus JR, Dechurch LA. Information sharing and team performance: a meta-analysis. J Appl Psychol. 2009;94:535-46.

22. Okuyama A, Wagner C, Bijnen B. Speaking up for patient safety by hospital-based health care professionals: a literature review. BMC Health Serv Res. 2014;14:61.

23. Pian-Smith MC, Simon R, Minehart RD, Podraza M, Rudolph J, Walzer $\mathrm{T}$, et al. Teaching residents the two-challenge rule: a simulation-based approach to improve education and patient safety. Simul. Healthc. 2009;4:84-91.

24. Reader T, Flin R, Lauche K, Cuthbertson BH. Non-technical skills in the intensive care unit. Br J Anaesth. 2006;96:551-9.

25. Sayre MM, McNeese-Smith D, Leach LS, Phillips LR. An educational intervention to increase "speaking-up" behaviors in nurses and improve patient safety. J Nurs Care Qual. 2012;27:154-60.

26. Kalisch BJ, Weaver SJ, Salas E. What does nursing teamwork look like? A qualitative study. J Nurs Care Qual. 2009;24:298-307. 
27. Wright MC, Phillips-Bute BG, Petrusa ER, Griffin KL, Hobbs GW, Taekman JM. Assessing teamwork in medical education and practice: relating behavioural teamwork ratings and clinical performance. Med Teach. 2009;31:30-8.

28. Guise JM, Segel S. Teamwork in obstetric critical care. Best Pract Res Clin Obstet Gynaecol. 2008;22:937-51.

29. Shetty P, Cohen T, Patel B, Patel VL. The cognitive basis of effective team performance: features of failure and success in simulated cardiac resuscitation. AMIA Annu Symp Proc AMIA Symp . 2009;2009:599-603.

30. De Dreu CK, Weingart LR. Task versus relationship conflict, team performance, and team member satisfaction: a meta-analysis. J Appl Psychol. 2003;88:741-9.

31. Gladstein DL. Groups in context: a model of task group effectiveness. Adm Sci Q. 1984;29:499-517.

32. Leasure EL, Jones RR, Meade LB, Sanger MI, Thomas KG, Tilden VP, et al. There is no "i" in teamwork in the patientcentered medical home: defining teamwork competencies for academic practice. Acad Med. 2013;88:585-92.
33. Manser T. Teamwork and patient safety in dynamic domains of healthcare: a review of the literature. Acta Anaesthesiol Scand. 2009;53:143-51.

34. Patterson PD, Weaver MD, Weaver SJ, Rosen MA, Todorova G, Weingart LR, et al. Measuring teamwork and conflict among emergency medical technician personnel. Prehosp Emerg Care. 2012;16:98-108.

35. Williams AL, Lasky RE, Dannemiller JL, Andrei AM, Thomas EJ. Teamwork behaviours and errors during neonatal resuscitation. Qual Saf Health Care. 2010;19:60-4.

36. Riskin A, Erez A, Foulk TA, Kugelman A, Gover A, Shoris I, et al. The impact of rudeness on medical team performance: a randomized trial. Pediatrics. 2015;136:487-95.

37. Azoulay E, Timsit JF, Sprung CL, Soares M, Rusinova K, Lafabrie A, et al. Prevalence and factors of intensive care unit conflicts: the conflicus study. Am J Respir Crit Care Med. 2009;180:853-60.

38. Sharma S, Boet S, Kito S, Reves S. Interprofessional simulated learning: the need for 'sociological fidelity'. J Interprof Care. 2011;25:81-3. 